\title{
Quelques réflexions sur l'histoire de ce qu'on dénomme exercices en didactique des langues
}

Some reflections on the history of what we call exercises in language teaching

\section{Henri Besse}

\section{(2) OpenEdition \\ 12 Journals}

\section{Édition électronique}

URL : https://journals.openedition.org/dhfles/6169

DOI : $10.4000 /$ dhfles.6169

ISSN : 2221-4038

\section{Éditeur}

Société Internationale pour l'Histoire du Français Langue Étrangère ou Seconde

\section{Édition imprimée}

Date de publication : 1 décembre 2019

Pagination : 43-67

ISBN : 0992-7654

ISSN : 0992-7654

\section{Référence électronique}

Henri Besse, «Quelques réflexions sur l'histoire de ce qu'on dénomme exercices en didactique des langues ", Documents pour I'histoire du français langue étrangère ou seconde [En ligne], 62-63 | 2019, mis en ligne le 12 avril 2020, consulté le 27 mars 2023. URL : http://journals.openedition.org/dhfles/6169 ; DOI : https://doi.org/10.4000/dhfles.6169

Ce document a été généré automatiquement le 27 mars 2023.

Tous droits réservés 


\title{
Quelques réflexions sur l'histoire de ce qu'on dénomme exercices en didactique des langues
}

Some reflections on the history of what we call exercises in language teaching

\author{
Henri Besse
}

Présentés comme étant (nous soulignons) « aujourd'hui une composante obligée » dans l'enseignement/apprentissage des langues (L1 ou L2), comme une « évidence " pour qui pratique ou étudie ce domaine, les exercices ne sont pas précisément définis par l'argumentaire de ce colloque. Quelques indices de ce qui est "traditionnellement rangé aujourd'hui sous l'étiquette d'exercices" permettent cependant de préciser quelque peu ce qu'on y entend par là.

Les exercices seraient une "forme " particulière des "activités de systématisation " propres à cet enseigner/apprendre, résultant d'une "construction pédagogique relativement récente ", d'une "invention collective » datable de la fin du XVIII ${ }^{e}$ siècle (il y est question de Meidinger, auteur d'une Praktische Französische Grammatik publiée en 1783) ou du début du XIX siècle (on y parle des cacologies et cacographies qui étaient alors en vogue), invention qui serait à l'origine de ces « recueils d'exercices » que les élèves peuvent exécuter hors de la classe.

Notre hypothèse de départ est que, sous-jacente à cet argumentaire, il y a une conception de ce type d'exercices qui est assez précisément datable et située. Elle est relative à une langue particulière, à savoir le français enseigné/appris en tant que L1 ou L2, et elle a été élaborée durant les années où l'étude théorisante de cet enseigner/ apprendre est passé, au moins en France, de la linguistique appliquée à la didactique des langues. Une conception qui n'était pas alors la seule mais que privilégie néanmoins ce colloque. 


\section{Deux conceptions des exercice(s) dont la formulation remonte au début des années 1980}

Pour ce qui est donc de la France, ces deux conceptions remontent à trois publications : un numéro des Études de Linguistique Appliquée [ELA] intitulé L'exercice $(1982,48)$ et deux ouvrages parus la même année, en 1984, qui se réfèrent à ce numéro mais qui s'opposent quant à la lecture qu'ils en font : L'exercice dans la classe de français de Gérard Vigner $^{1}$; Grammaires et didactique des langues d'Henri Besse \& Rémy Porquier.

\section{Une conception focalisée sur l'enseignement plus que sur l'apprentissage}

Selon sa quatrième de couverture, l'ouvrage de Vigner se proposait « de définir [...] les propriétés constitutives de l'exercice » et «à quelles conditions une activité mérite [...] le nom d'exercice ». Projet qui écartait « la pédagogie des langues maternelles de [son] champ d'investigation " (Vigner 1984:18) et dont la définition était éprouvée sur un échantillon d'exercices relevant d'une quarantaine de manuels de français L2, publiés par des éditeurs parisiens entre 1955 et 1982.

Pas moins de cinq pages (ibid.: 13-18) tentent d'y "préciser une notion dont la définition fait problème", parce qu'elle s'inscrit «dans un réseau complexe d'oppositions » dont les trois suivantes : a) "si tout exercice est bien une activité langagière, [...] toute activité langagière ne saurait être assimilée à un exercice ", celuici supposant «l'existence d'un objectif précis »; b) l'exercice est « une tâche » qui doit être exécutée « en un temps restreint ", être "réitérée un nombre important de fois " et viser à "la seule mise en œuvre d'habitudes ou d'automatismes", alors que la résolution de problème "exige de l'apprenant la mobilisation de l'ensemble de ses acquis et de ses capacités » et fait confiance à sa « libre initiative »; c) dans les manuels et les classes, on distingue traditionnellement "la leçon", qui vise à enseigner des connaissances nouvelles, des « séries d'exercices » qui en sont la "phase d'utilisation ou d'application ».

Suit une définition d'une vingtaine de lignes (ibid.: 17) dont nous retenons qu'il s'agit "d'une tâche imposée ou proposée à l'apprenant » par le formateur (l'enseignant ou le manuel), qui porte "sur une composante ou difficulté de l'apprentissage ", qui est "répétitive, un nombre minimum d'essais [étant] prévu», qui exige des «délais de réponse brefs » et dont la «variabilité tolérée dans la forme de la réponse » est « faible ou nulle ", tâche débouchant «sur une réponse observable et aisément évaluable, par l'apprenant comme par le formateur ».

Quels que soient les mérites de cette définition, son abstraction et la complexité des oppositions qu'elle met en jeu font que c'est à l'application qu'en fait Vigner dans l'analyse de son échantillon d'exercices, qu'on saisit le mieux le sens pour ainsi dire prototypique qu'il prêtait alors au mot exercice(s).

Par exemple, à propos de ce que nous avions étiqueté « exercices de conceptualisation » dès 1974, Vigner dit qu'ils ne sauraient être « envisagés autrement que comme activité de classe, par opposition aux exercices habituels qui peuvent être effectués individuellement, hors de tout contexte institutionnel » (ibid.: 94), ajoutant que «le terme d'exercices, stricto sensu, n'est d'ailleurs peut-être pas adéquat» pour une 
activité qui est proche de la "résolution de problème" (ibid.: 95). À propos des «nouveaux exercices » apparus avec les approches communicatives, Vigner écrit qu'ils rompent avec une "certaine orthodoxie pédagogique » entendue ainsi : "des tâches " limitées aux phrases, se refusant à « recourir à une explicitation verbale des règles » et engageant «un apprentissage conduit selon les principes d'inspiration nettement behavioriste » (ibid. : 126). Ce qui fait que, pour ces pratiques communicatives aussi, «le terme d'exercice ne convient pas exactement» (ibid.). Indices, parmi bien d'autres, d'une " orthodoxie pédagogique » tributaire des exercices structuraux, tels qu'ils ont été conçus et pratiqués en France de la fin des années 1950 à la fin des années 1970.

L'emprise de cette "orthodoxie » est particulièrement nette à propos de l'ouvrage d'Elizabet Hammar sur l'apprentissage du français en Suède aux XVII ${ }^{e}$ et XVIII ${ }^{e}$ siècles, dont Vigner dit qu'elle y dénomme « improprement » (ibid. : 38) exercices toutes " sortes d'activités » (conversations, dialogues, proverbes, maximes, courtes histoires ou divers travaux écrits). Tout en admettant, à propos de quelques «tableaux » que Chevalier (1968: 401-404) avait repris de la Briefve Institution de Du Vivier "publiée en 1568 à Cologne ", que " de tels tableaux à nos modernes exercices de substitution il n'y a qu'un pas à franchir » (ibid. : 40). Tout comme il admet que les exercices extraits par Charles Bouton (1972) des « grammaires françaises de Claude Mauger à l'usage des Anglais » du $\mathrm{XVII}^{\mathrm{e}}$ siècle sont proches de ces mêmes exercices "de substitution » (ibid.: 41 et 42). Il n'en conclut pas moins (nous soulignons) que, «à ces tendances près, nous ne sommes pas encore en présence d'exercices au sens où nous l'entendons aujourd'hui » (ibid.).

Il y a là, nous semble-t-il, un parti pris que Vigner justifie en s'appuyant moins sur l'histoire de l'enseignement/apprentissage du français L2 que sur celui du français L1 (nous soulignons): "en français langue maternelle s'instaure l'usage de l'exercice qui trouve désormais sa place dans les ouvrages de grammaire, sous l'influence de Noël et Chapsal » (ibid. : 42). Ce qu'il étaie par trois phrases de la thèse d'André Chervel (1977) : «Avec l'apparition d'exercices annexes, c'est le statut de la grammaire qui change. On consultait la grammaire pour apprendre, ou comprendre, la langue. On va désormais se livrer à ces exercices pour apprendre la grammaire. » Double référence à « la pédagogie de langue maternelle ", ou supposée telle, que Vigner avait pourtant (voir ci-dessus en début de cette sous-partie) écartée. Jean Hébrard, directeur du $n^{\circ} 48$ des ELA, y a publié un article intitulé: «L'exercice de français est-il né en $1823^{2}$ ?». La "certaine orthodoxie pédagogique » dont se réclamait Vigner en 1984 revient à ignorer ce point d'interrogation.

\section{Une conception focalisée sur l'apprentissage plus que sur l'enseignement}

L'ouvrage de Vigner (1984) et Grammaires et didactique des langues ${ }^{3}$ sont parus la même année et n'ont donc pu mutuellement s'influencer. Mais nous y citions Vigner (1982) au début de notre chapitre 6 et nombre de nos références sont communes, d'où il résulte que la conception de nos « exercices grammaticaux » est à la fois proche et différente de la sienne ${ }^{4}$.

Un seul exemple (nos actuelles italiques) : «En résumé, un exercice pourrait être défini comme une tâche langagière ponctuelle à caractère répétitif, contraint et métalinguistique marqués, tâche demandée par le professeur aux étudiants et évaluée par lui.» (Besse \& Porquier 1984:121) Les mots sont quasi les mêmes que ceux de 
Vigner, mais les sens que nous leur avions préalablement donnés différaient sensiblement des siens.

Le caractère répétitif des exercices y était aussi privilégié, mais nous le qualifions de réitération et non de répétition: "Il n'y a pas exercice sans réitération, c'est-à-dire sans avoir à faire plusieurs fois la même chose» (ibid.: 120). Il ne s'agit donc plus du psittacisme d'une série de phrases supposées analogues d'un point de vue structural, mais de la réitération d'un faire langagier qui engage des activités autres que mimétiques. Vigner se voulait attentif au «matériel» de la répétition, toute activité «réglée par les propriétés du matériel » relevant à ses yeux de «la procédure de l'exercice » (1984: 150); nous l'étions davantage au sémantique et au conceptuel de la réitération ${ }^{5}$. Ponctuel y renvoie moins à l'objectif limité de tout exercice qu'à sa perte d'efficacité dès que les apprenants le trouvent trop long. Par contraint nous entendions certes, tout comme Vigner, « le caractère contraint de la réponse attendue : la tâche doit être exécutée selon des règles précisées par l'exercice (dans la consigne ou dans le modèle) », car « si la réponse n'est pas en partie préréglée par l'injonction de départ, on ne peut dire qu'il y a 'exercice'» (Besse \& Porquier 1984:120); mais un exercice avait aussi à nos yeux pour fin de fixer ou de corriger " par une certaine prise de conscience, ce qui est en cours d'apprentissage ", ce qui est bien peu béhavioriste (ibid.: 121). Quant à métalinguistique, Vigner l'entendait comme renvoyant au jargon des grammairiens et des linguistes, alors que nous l'entendions différemment : l'exercice étant une " tâche fractionnée " qui porte "sur une composante ou une difficulté [...] isolée de sa complexité originelle ", il est inévitablement focalisé « non pas sur le monde mais sur le langage ", ce qui le rend, "essentiellement métalinguistique » (ibid.: 120-121), y compris quand le jargon grammatical ou linguistique en est absent.

L'évidence qu'un exercice est une «tâche demandée par le professeur aux étudiants et évaluée par lui » (ibid.) n'en était pas, non plus, tout à fait une pour nous, tout exercice s'inscrivant à nos yeux dans un "rituel communicatif » ternaire: "l'exercice est un texte ou un discours produit par le maître et visant à proposer ou à imposer aux étudiants une tâche langagière précise (premier temps de l'échange); cette tâche est effectuée par ceux-ci, en réponse à l'injonction magistrale (second temps); le maître porte un jugement sur la qualité de la tâche (troisième temps)»(ibid.). Et nous ajoutions que, de ce rituel, «seul le premier temps apparaît dans les manuels, mais tant qu'il n'y a pas eu effectuation de l'exercice par l'étudiant et correction par le maître, on ne peut considérer que l'exercice est achevé : l'apprenant conserve l'impression que l'échange n'est pas clos » (ibid.).

En d'autres termes, l'exercice tel qu'il est observable dans un manuel de L2 n'est pas l'exercice tel qu'on peut l'observer dans une classe utilisant ce manuel, et encore moins tel qu'il est effectivement pratiqué par celles ou ceux qui, dans leur diversité cognitive (émotionnelle et conceptuelle), l'exécutent ${ }^{6}$. D'où ce qui était, et est toujours, une autre évidence à nos yeux: l'efficacité d'un exercice dépend moins des théories psychologiques ou linguistiques qu'il est censé appliquer - présupposé dominant de la linguistique appliquée des années 1950-1970 - que de ce que chaque enseignant et surtout chaque enseigné en fait dans le hic et nunc de leurs interactions au sein du groupe-classe. L'ouvrage de Vigner est certes titré L'exercice dans la classe de français, mais la classe, où les exercices sont effectivement mis en œuvre pour enseigner/ apprendre une L2, n'y est guère présente ${ }^{7}$. 
Pourtant, selon que l'on est attentif ou non à ce qui se passe effectivement dans une classe quand un exercice y est pratiqué, qu'on suppute ou non ce qui a pu se passer dans les classes du passé, on analyse différemment cet exercice. Si l'on se borne à ce qu'est un exercice dans un manuel, on privilégie le côté méthodologique de l'exercice ${ }^{8}$; si l'on tient compte des façons dont les enseignants le mettent (ou peuvent le mettre) en pratique en classe, des réactions, avérées ou supputées, des apprenants qui l'exécutent, l'analyse qu'on en fait tend à privilégier ce qu'il est ou peut être effectivement pour ceux (celles) qui l'exécutent. C'est pourquoi notre typologie des «exercices grammaticaux", se voulait «fondée essentiellement sur le 'travail demandé" aux étudiants, la tâche qu'ils doivent accomplir " (Besse \& Porquier 1984: 124). Ce qui en excluait certains jeux de langage ou de rôles, «non contraints dans leur contenu linguistique " (ibid.); et certaines tâches consistant par exemple à confectionner un objet à partir de sa notice de montage ou un plat à partir de sa recette.

Nous nous en tenions, dans notre chapitre 6, à quatre "grands types d'exercices", ceux «de répétition», ceux «à trous", les «structurels " et ceux «de reformulation», auxquels il faut ajouter nos exercices de conceptualisation dont traitait la dernière partie de notre chapitre 5, intitulée "La perception métalinguistique de l'apprenant » (ibid. : 109-115). De ces "cinq grands types", Vigner (1984) n'en retenait à peu près qu'un seul, celui dit « structurel ». Et dans l'analyse que nous en proposions, notre souci de la diversité de celles et de ceux (maîtres et élèves) susceptibles de les mettre en œuvre en classe était constant. Pour les exercices de répétition, nous recommandions « une pédagogie de la perception, auditive bien entendu, mais aussi grammaticale, sémantique et communicative ", l'apprenant répétant "mal parce qu'il entend mal, dans les deux sens qu'entendre peut avoir en français: 'percevoir par l'oreille' et 'percevoir par la pensée' $\aleph^{10}$ (ibid.). Pour les exercices à trous, nous disions que ce qui en "détermine les 'bonnes réponses'", c'est moins leurs consignes, que leurs "présupposés contextuels", lesquels ne coïncident que rarement avec ceux mobilisés spontanément par les apprenants (ibid.: 129). Pour ceux dits «structurels» (qu'il s'agisse des exercices structuraux, des exercices de réemploi, des micro-conversations ou de certains exercices « communicatifs »), nous notions que, non contextualisés, leur psittacisme incite les apprenants à des " généralisations abusives » en L2 (ibid. : 135), et lorsqu'ils le sont peu ou prou, leurs mini-contextes modifient "souvent la structure qu'on cherche à (faire) réitérer en l'affectant de modalités, d'aspects, de valeurs qui, pour ne pas être apparentes, ne la déterminent pas moins grammaticalement » (ibid.: 133). Pour ceux que nous disions «de reformulation » (ibid.: 142-144), fondés sur une "caractéristique fondamentale» des langues naturelles qui permet de dire «de plusieurs façons 'la même chose'» (ibid.: 126), nous en donnions un seul exemple, emprunté à une classe de Marc Argaud et Benoit Marin (1972) où ils utilisaient le manuel De vive voix ${ }^{11}$. Quant à nos «exercices de conceptualisation" (ibid.: 109-115), il s'agit d'une transposition didactique de l'épistémologie grammaticale développée dans les trois premiers chapitres de Besse \& Porquier 1984, où il est montré, au moyen d'exemples plus que par un discours abstrait, que les règles des grammairiens et des linguistes sont des artefacts et non les « règles " qui permettent à un natif de parler sa L1 comme elle est parlée ${ }^{12}$, et encore moins les "règles " permettant d'enseigner/ apprendre efficacement une L2. Et pour ces cinq types d'exercices, nous proposions un «bon usage» qui consistait, pour l'enseignant, à évaluer ce que l'apprenant fait individuellement d'un exercice non en fonction de la « bonne réponse » attendue ${ }^{13}$ mais 
en fonction de ce que cet exercice lui donne l'occasion de dire, compte tenu de " l'interlangue » à laquelle il est parvenu, dans la L2 qu'il est en train de s'approprier.

$\mathrm{Au}$ début des trois pages (ibid.: 113-115) consacrées à nos exercices de conceptualisation, nous précisions (nos actuelles italiques): "il s'agit moins, ’̀ proprement parler, d'exercices que de moments de réflexion grammaticale explicitée par les apprenants", et nous concluions ces trois pages ainsi: "un exercice de conceptualisation n'est jamais reproductible à l'identique, et c'est en ceci qu'il n'est pas un exercice: les corpus de départ changent selon les classes, [...], et les hypothèses 'explicatives' peuvent varier considérablement dans leur métalangage comme dans leurs conceptions".

Ce qui revenait à admettre qu'en didactique du français (L1 ou L2), il y avait déjà en 1984 deux conceptions opposées de ce que peut-être un exercice : une restreinte telle qu'entendue par Vigner et Hébrard (1982) à propos du français L1 plus que du français L2, qui est focalisée sur l'enseignement plus que sur l'apprentissage ; une plus extensive telle que nous en usions depuis les années 1970 à propos du français L2 plus que du français L1, focalisée sur l'apprentissage plus que sur l'enseignement. Une conception de l'exercice qui ne faisait que reprendre celles des historiens de l'enseignement des langues (L1 ou L2), entre autres Jean-Claude Chevalier (1968), pour ce qui est des emplois d'exercice(s), et Louis G. Kelly (1976) pour ce qui est de ses équivalents anglais (exercises, grammar drills, pattern practice). Les « colloques» de Mathurin Cordier (1586) et les «sentences» de William Bathe dans sa Janua lingvarum (1611) n'étaient-ils pas qualifiés par le premier « d'exercices qui, en un sens, rendent inutiles les grammaires, inutile l'apprentissage fastidieux de règles multiples et barbares", y voyant, tout comme Chervel (voir ci-dessus la citation qu'en fait Vigner), "un substitut de la grammaire » (1968: 383) ? Les Colloquia [1524] d'Érasme n'étaient-ils pas qualifiés par le second de pattern practices? Bref, nous usions d'exercice(s) tout comme l'avait fait Hammar, « improprement » selon Vigner.

C'est que le projet de Vigner était différent du nôtre en cette même année 1984. Le sien était d'expliciter le modèle alors dominant dans les manuels de français L2 publiés par les éditeurs parisiens, afin d'aider les auteurs de manuels et les enseignants de cette langue à "construire des exercices» par eux-mêmes, selon l'intitulé du dernier chapitre de son ouvrage (1984:193-210), un projet partant focalisé sur l'enseignement plus que sur l'apprentissage. Le nôtre était d'intégrer les exercices - en particulier ceux des manuels d'inspiration $\mathrm{SGAV}^{14}$ - dans une réflexion d'ordre épistémologique portant sur l'enseignement/apprentissage de la "grammaire" du français L2, d'où notre attention à leur «bon usage » dans le hic et nunc des classes. Projet plus « linguistique appliquée » pour Vigner, plus « didactique » pour Besse \& Porquier.

Vigner a publié fin 2017 un ouvrage, sous-titré l'exercice en FLE, où nos divergences de 1984 sont atténuées. On y retrouve in extenso (Vigner 2017: 16) sa définition de l'exercice tel qu'il l'entendait alors mais aussi (ibid.: 11) la nôtre de 1984, laquelle est toutefois amputée du «En résumé » qui l'ouvrait et qui renvoyait à ce que nous avions préalablement précisé quant à ponctuel, caractère répétitif, métalinguistique ${ }^{15}$ ou réitération ${ }^{16}$. Il y est, en outre, question de "familles d'exercice(s) ", ce qui le rapproche de nos «types d'exercices ». Il y est moins soucieux d'aider les auteurs de manuels et les enseignants de français L2 à « construire des exercices », et plus attentif à ce qu'il advient de ces exercices quand ils sont pratiqués en classe (voir entre autres, ibid. 78-79: « L'exercice dans le déroulement de l'apprentissage »). 
Mais quant aux «origines de l'exercice " (titre de son chap. 2), il s'en tient à ses convictions de 1984, celles qui sous-tendent l'argumentaire du présent colloque: «l'exercice [...] est apparu de façon relativement récente, dans la fin du XVIII siècle" (ibid.: 30); Meidinger en fut l'un de ses principaux promoteurs (ibid.: $32)$; «tout au long du XIX siècle » se sont développées diverses formes d'exercices, dont les cacographies (ibid.: 32 ); et "l'exercice structural, introduit au milieu du $\mathrm{XX}^{\mathrm{e}}$ siècle " $\mathrm{y}$ est posé comme "une étape nouvelle, tout à la fois dans le mode d'organisation de l'activité et dans la relation de l'élève à la langue » (ibid.: 36). S'y retrouve aussi la citation de Chervel interprétée, à la manière de Chevalier (1968) caractérisant les «colloques» de Cordier et les «sentences» de Bathe, comme des «exercices qui [...] rendent inutiles les grammaires», citation sur laquelle nous allons revenir après avoir exploré l'histoire du mot exercice(s) quant à ses emplois en matière d'enseigner/apprendre les langues (L1 ou L2).

\section{Quelques jalons relatifs à I'histoire du mot exercice(s)}

Cette réflexion s'appuie sur l'étymologie du mot français exercice et sur ce que les dictionnaires "généraux» de cette langue en ont dit depuis qu'ils existent. Deux savoirs relativement incertains, mais à même de contribuer, de manière au moins heuristique, à notre réflexion.

\section{Sur l'étymologie d'exercice(s)}

Les dictionnaires étymologiques actuels font de ce substantif français un calque, attesté dès le XIII ${ }^{e}$ siècle, du latin exercitium, dérivé nominal du verbe exercere. Et il doit en aller peu ou prou de même, à l'exception peut-être de la date de leurs premières attestations, pour les équivalents littéraux d'exercice dans nombre de langues européennes : exercise en anglais; ejercicio en espagnol ; esercizio en italien. L'allemand use certes du verbe exerzieren ("faire l'exercice », " manœuvrer ») mais le confine aux choses militaires, et son dérivé nominal semble inusité ; dans l'étude des langues, on use de Übung d'origine germanique (du verbe üben, " entraîner, exercer, pratiquer »).

Dans le monde de la latinité, tant le grammatiste (celui qui enseignait à lire et à écrire les lettres) que le grammairien (celui qui enseignait la «science » de la grammaire) n'utilisaient guère, semble-t-il, le substantif exercitium pour dénommer les pratiques de classe destinées à préparer leurs élèves à l'enseignement du rhéteur (celui qui enseignait la «science » de la rhétorique). Ils usaient du mot grec progymnasmata, son équivalent littéral latin praeexercitamina n'étant pas attesté avant Priscien ( $\mathrm{VI}^{\mathrm{e}}$ siècle). C'est progymnasmata qu'on trouve dans le Liber primus du De institutione oratoria de Quintilien (fin du I $^{\mathrm{er}}$ siècle après J.-C.) ou dans le titre d'un recueil d'Hermogène de Tarse (fin du II ${ }^{\mathrm{e}}$-début du $\mathrm{III}^{\mathrm{e}}$ siècles) que Priscien a latinisé en praeexercitamina. Le succès qu'ont connu les Institutiones grammaticae de ce dernier est sans doute pour beaucoup dans les différents calques que les langues européennes de l'Ouest ont faits d'exercitium.

Qu'entendait-on, dans le monde gréco-latin des six premiers siècles de notre ère, par progymnasmata? À s'en tenir à ce qu'en dit Quintilien dans le chapitre 9 de son premier livre, ce sont des " exercices préparatoires à l'expression $»^{17}(\mathrm{I}, 9,1)$ consistant soit à apprendre à dire oralement dans un « langage pur» des fables d'Ésope (qui, étant en 
prose, n'étaient pas à réciter) avant de " les mettre par écrit ", soit à « rompre les vers " des poèmes et à $\mathrm{y}$ "remplacer les mots par des équivalents, puis à procéder à une paraphrase plus libre [...] tout en respectant la pensée du poète » $(\mathrm{I}, 9,2)$, soit à dire des «aphorismes [sententiae], des chries [chria], des étiologies [aetiologiae]» (I, 9, 3), soit encore à retenir, pour meubler leur mémoire, des « anecdotes [narratiuncula] rendues célèbres par les poètes » $(I, 9,6)$. Autant d'exercices préparatoires à l'expression plus orale qu'écrite, qui ne sont pas sans analogie avec nos exercices de reformulation voire de conceptualisation ${ }^{18}$.

\section{De l'histoire du mot exercice(s) d'après les dictionnaires de la langue française}

Bien que les formes exercice ou exercite soient présentes depuis le XII ${ }^{e}$ siècle dans les manuscrits rédigés en français, elles ne semblent pas y avoir pris le sens «d'exercice scolaire pour apprendre une langue " avant les XVII ${ }^{e}$-XVIII ${ }^{e}$ siècles. Les dictionnaires philologiques qui ont recensé les usages de ces deux formes en bas et moyen français (du XII ${ }^{e}$ au XVI ${ }^{e}$ siècle) en attestent ${ }^{19}$. Sans doute parce que les langues, qu'elles soient dites savantes ou vulgaires (tel le français), étaient très généralement, au moins dans les collèges, enseignées en latin, et si l'on pratiquait en classe l'un ou l'autre des " exercices préparatoires à l'expression », on en parlait en usant du latin exercitium.

Le Thresor de la langue françoyse tant ancienne que moderne de Jean Nicot [1606 dans sa version alphabétique] est un bilingue, où exercice est traduit par trois mots latins: studium ("application zélée, zèle, dévouement, étude», selon le Gaffiot); subactio ("préparation, formation de l'esprit »); exercitium (" exercice, pratique », " exercice militaire »). Mais prêtait-on en 1606 à ces mots latins les sens que Gaffiot leur donne en 1934 ? Le Richelet [1680] définit exercer par «mettre en usage, ou en pratique quelque chose qu'on apprend, ou qu'on sait faire (s'exercer à la chasse, à étudier; exercer une charge, la médecine...)»; et exercice, par "action de la personne qui s'éxerce. Occupation ». Le Furetière [1690] glose exercice à peu près comme le Richelet, mais donne d'exercer une définition pour ainsi dire béhavioriste : «Faire prendre à quelqu'un une habitude pour le faire bien réussir à quelque chose ».

Dans la première édition du Dictionnaire de l'Académie [1694] exercice est entendu comme « l'action par laquelle on s'exerce » aux armes et à la guerre, mais on y trouve aussi quant à ses emplois au pluriel : "Se dit particulierement des diverses choses que les jeunes gens apprennent à l'Académie, comme monter à cheval, faire des armes, danser, voltiger, \&c. ». Ce n'est qu'à sa quatrième édition [1762] qu'on y trouve cet emploi «particulier »: " On appelle au Collége, Exercices, Certaines conférences où les écoliers répondent sur quelque partie des Humanités. ${ }^{20}$ Peut-on voir là un indice que les (prae)exercitamina de Priscien sont désormais appelés, au moins parfois en français, des exercices?

On sait que, dans certains collèges jésuites d'Espagne ou d'Italie de la seconde moitié du $\mathrm{XVII}^{\mathrm{e}}$ siècle, on enseignait le français comme un "art chevaleresque " à l'égal de l'escrime, l'équitation ou la danse. Peut-être y usait-on déjà d'exercices, d'ejercicio ou d'esercizio en matière d'enseignement du français L2. On trouve dans les premières pages du manuel de Nicolas Chantreau [1781], francophone d'origine, un Exercicio diario que debe hacer el discipulo para perfecccionarse en la pronunciacion del francés, exercice 
proche, on en conviendra, des exercices "d'aujourd'hui » tels qu'entendus par l'argumentaire de ce colloque ${ }^{21}$.

\section{Du mot exercice(s) dans l'Encyclopédie de d'Alembert et Diderot}

Les emplois d'exercice(s) ${ }^{22}$ dans l'Encyclopédie sont d'autant plus révélateurs des usages de ce mot au milieu du XVIII ${ }^{e}$ siècle qu'ils sont venus spontanément, au moins pour ceux qui nous intéressent ici, sous la plume de contributeurs qui ne cherchaient pas à en élaborer une définition.

On y trouve une entrée Progymanasmata, catégorisée Gymnastique (t. $13: 347)$ : « 'nom qu'on donnait aux exercices préparatoires que devaient faire tous ceux qui se présentaient pour disputer les prix dans les jeux olympiques ${ }^{23}$ ». Exercitium n'y a pas d'entrée, mais on en trouve deux occurrences : une à l'entrée Chymie ou Chimie et une à l'entrée Milice, catégorisée Art militaire, sous-entrée " discipline des Romains» (t. 10 : 511) : « Les exercices militaires faisoient une autre partie de la discipline ; aussi c'est du mot exercitium, exercice, que vient celui d'exercitus, armée, parce que plus des troupes sont exercées, plus elles sont aguerries. " On n'y trouve donc rien concernant l'enseignement/apprentissage des langues, tout comme à l'entrée Exercice (t. 6: 238-252), catégorisée Art militaire, et à ses sous-entrées (Marine, Hygiène-Médecine, Manège). Quant à l'expression exercices $d u$ corps \& de l'esprit, elle est assez répandue dans l'Encyclopédie, mais esprit y renvoie le plus souvent au " spirituel, religieux ».

Si l'on consulte les entrées rédigées par des contributeurs qui se sont intéressés à l'enseignement/apprentissage des langues, certaines ne contiennent aucune occurrence d'exercice(s) au sens où l'entend ce colloque ${ }^{24}$, alors que d'autres attestent d'emplois plus ou moins conformes tant à nos propres emplois de 1984 qu'à ceux dits «d'aujourd'hui» dans son argumentaire. Bornons-nous à quelques-unes de ces occurrences (nous soulignons exercices).

À l'entrée Classe (t. $3: 506$ ), Dumarsais conseille, lorsque « l'éducation domestique » est "trop solitaire \& trop languissante ", de faire "souvent des assemblées, des exercices, des déclamations ", ce qui suggère qu'à ses yeux les exercices relèvent du collectif, tout comme les « conférences » dont il est question dans l'édition de 1762 du Dictionnaire de l'Académie. À l'entrée Études, Faiguet de Villeneuve précise qu'on désigne par ce mot l'ensemble des "exercices littéraires usités dans l'instruction de la jeunesse, études grammaticales, études de Droit, études de Médecine» (t. $6: 87$ ), avant de critiquer un " exercice littéraire " pratiqué dans « les meilleurs collèges » consistant, « depuis sept à huit ans jusqu'à seize \& davantage ", à se former «à la composition du latin » par des «themes », exercice dont les élèves ne recueillent « aucun fruit réel ». Faiguet préfère suivre la méthode de «M. Le Febvre » où le « même temps » est donné « à l'explication des auteurs \& aux autres exercices» telle la "version» (ibid.: 88), qui sont «moins difficiles \& moins rebutans que des themes " (ibid.: 91). Ou encore à l'entrée Faculté, catégorisée Physiologie, Jaucourt écrit, pour justifier la supériorité des hommes sur les animaux, qu'on «s'en [de cette supériorité] apperçoit facilement même dans les bêtes les plus dociles, lorsqu'on leur apprend quelques exercices, puisque ce n'est que par une longue suite d'actes répétés, qu'on peut les former à ces exercices » (t. $6: 364)$, ce qui sous-entend que les hommes peuvent être dispensés de ces fastidieuses réitérations.

Et l'on pourrait citer bien d'autres occurrences d'exercice(s) dans l'Encyclopédie pris en un sens proche de ce que nous entendons encore par ce mot en matière d'enseigner/ 
apprendre les langues, tels les exercices de thème et de version absents de l'argumentaire de ce colloque. Même si le Dictionnaire de l'Académie ne reconnaît cet emploi pluriel qu'en 1762, il nous semble avéré qu'il s'était répandu bien avant, au moins dans la langue des maîtres et des élèves des collèges de France.

\section{L'entrée exercice dans le dictionnaire d'Émile Littré}

Pour le XIX ${ }^{\mathrm{e}}$ siècle, nous nous en tiendrons à un seul dictionnaire, celui d'Émile Littré [1873-1874, en 4 vol.]. Entre autres raisons, parce que son entrée Exercice nous paraît à la fois récapituler et compléter ce que nous venons d'avancer (de 2.1. à 2.3.), en suivant des voies en partie différentes des nôtres.

Cette entrée exercice, que Littré dérive d'exercitium, distingue quinze sens différents, du plus «simple et primitif» ( $1^{\circ}$ Action d'exercer quelqu'un à quelque chose ou de s'y former soi-même. Cela ne s'apprend que par un long exercice.») au plus «métaphorique " ( $15^{\circ}$ Visite des commis chez certains marchands pour la perception des droits indirects. $»)^{25}$. Le sens qui nous intéresse ici est le $7^{\circ}$, que nous citons dans son intégralité (à l'exception de ses deux exemples) :

$7^{\circ}$ Terme de collége. Conférences où les écoliers répondent sur certaines parties des humanités. Soutenir un exercice. Exercices publics, conférences qui se faisaient dans les hautes écoles. [un exemple tiré de Fléchier et un autre de Fontenelle] Dans les classes, exercices au tableau, les exercices de traduction ou de calcul que l'élève fait à la craie sur un tableau noir, sous la direction du professeur. Il se dit aussi des devoirs ou compositions donnés pour familiariser l'élève avec les règles. Les thèmes, les versions, les analyses sont d'excellents exercices pour apprendre les langues. Il se dit enfin des livres qui contiennent les exercices.

Définition en extension qui reprend les éditions du Dictionnaire de l'Académie depuis 1762 , mais qui y ajoute des emplois en usage « dans les classes » de langues et de calcul. Emplois qui ne sont guère éloignés de nos propres emplois, même s'ils ne se réfèrent pas aux mêmes exercices. Encore que le «se dit aussi des devoirs ou compositions donnés pour familiariser l'élève avec les règles » peut y renvoyer.

\section{Les exercices dans deux dictionnaires récents spécialisés en didactique du français $L 2$}

Tenons-nous en au Robert Galisson \& Daniel Coste (1976) et au Jean-Pierre Cuq (2003).

Du premier - antérieur donc à la réflexion sur l'exercice (au singulier générique) du début des années 1980 (voir notre première partie) -, notons qu'il définit Exercice plus « en psychologie », telle qu'elle « est généralement admise par toutes les théories » de cette science ${ }^{26}$, qu'en "didactique des langues", où se retrouve plus ou moins la définition des dictionnaires non spécialisés : l'exercice a " pour but l'assimilation et la mise en place fonctionnelle des éléments linguistiques antérieurement présentés et expliqués » et "par extension", le terme s'applique "au matériel pédagogique et linguistique servant de base et de prétexte à l'activité de l'élève», tel "un livre d'exercices, une batterie d'exercices». Deux types en sont distingués: les "exercices dits 'systématiques' » (avec renvoi aux entrées Drill et Structural 3), et ceux «moins formels et moins contraignants, dits de 'réemploi' ou d'exploitation'». Allusion à la rivalité entre les tenants du SGAV et les tenants de l'audio-oral nord-américain. L'entrée Drill précise que «le sens originel de ce mot anglais évoque l'entraînement 
physique (militaire ou sportif) », et qu'il s'agit « d'une sorte de gymnastique mentale et motrice qui doit conduire au maniement automatique de formes linguistiques précédemment comprises ». Entrée rédigée une dizaine d'années avant Vigner (1984) qui se conclut déjà ainsi : «Les principes qui ont fondé la pratique des drills sont aujourd'hui très controversés. »

Le dictionnaire dirigé par Cuq consacre (2003: 94-97) pas moins de neuf entrées à la notion d'exercice, une se voulant générale (Exercice) et huit plus spécifiques, traitant des exercices dits "à trous ", "de réemploi ", « de reformulation ", "de répétition ", " de substitution », « de suppression », « de transformation » et «structural ». L'entrée générale renvoie à pas moins de douze entrées (d'Activité à Traduction, en passant par Jeu de rôle, Question à choix multiples ou Simulation); l'entrée Exercice structural, aux quatre qui la précèdent (Exercice, E. de répétition, E. de substitution, E. de transformation) mais aussi à huit autres (allant d'Audio-oral à Syntagmatique, en passant par Béhaviorisme, Laboratoire ou Structure).

Nous ne pouvons ici nous livrer à une analyse critique de toutes ces entrées. Bornonsnous au début de l'entrée Exercice dont le rédacteur n'est pas nommé :

Bien que ce terme soit souvent employé au même sens qu'activité d'apprentissage,

l'exercice renvoie à un travail méthodique, formel, systématique, homogène, ciblé vers un objectif spécifique. Au sein d'un ensemble construit d'activités, l'exercice est conçu pour répondre à une difficulté particulière. Même si, par ses origines historiques, le terme est souvent relié au travail grammatical, on peut l'utiliser pour désigner l'ensemble des travaux d'apprentissage linguistique et communicatif.

On y débute par une concessive (introduite par bien que) qui reprend, sous des mots de sens voisin, la première des trois oppositions (« toute activité langagière ne saurait être assimilée à un exercice») structurant selon Vigner (1984) la notion d'exercice. L'exercice ne serait pas une "activité d'apprentissage » comme les autres en raison de ses aspects "méthodique, formel, systématique, homogène, ciblé vers un objectif spécifique " ou "une difficulté particulière ». Et elle se poursuit par une seconde concessive (introduite par même si) admettant que cette notion, originellement liée au « travail grammatical », est néanmoins utilisée " pour désigner l'ensemble des travaux d'apprentissage linguistique et communicatif ». Deux concessives visant à avertir que cette définition d'exercice ne correspond pas aux emplois les plus courants de ce terme, et qu'elle est donc spécialisée. Quant aux deux autres oppositions structurant cette notion selon Vigner (1984), celle excluant la « résolution de problème » des exercices stricto sensu et celle opposant la leçon aux exercices, la suite de l'entrée Exercice du Cuq inclut quant à elle les « résolutions de problème » et on n'y trouve pas d'entrée Leçon, alors qu'on en trouve une dans le Galisson-Coste.

Quelle conclusion tirer de ces quelques jalons lexicographiques? Que les exercices, bien plus que le générique l'exercice, est en usage en français au moins depuis la fin du $\mathrm{XVII}$ e siècle pour "diverses choses que les jeunes gens apprennent à l'Académie, comme monter à cheval, faire des armes, danser, voltiger, \&c ${ }^{27}$; un et cetera qui pouvait sans doute inclure, dans certains collèges dès ce siècle mais plus encore au suivant, diverses tâches pour enseigner/apprendre les langues tant savantes que vulgaires, soit bien avant que la quatrième édition du Dictionnaire de l'Académie française [1762] ne l'admette en termes allusifs. Le terme s'applique alors, comme en attestent les emplois qu'en font spontanément une partie des rédacteurs de l'Encyclopédie s'intéressant à l'enseignement des langues, à des tâches menées 
collectivement en classe sur des questions de grammaire, de poésie ou de rhétorique, mais qu'on pouvait aussi mener individuellement en présence ou non d'un précepteur.

Parmi ces exercices, certains différaient certes de ceux dont il est question dans l'argumentaire de ce colloque, bien qu'ils fussent aussi qualifiables, tout comme l'Exercice selon le Cuq, de «travail méthodique, formel, systématique, homogène, ciblé vers un objectif spécifique». Parmi ceux dont il n'est pas question dans cet argumentaire, il y a la version et le thème dénommés exercices au moins depuis le $\mathrm{XVII}^{\mathrm{e}}$ siècle, et qui plus est ne sont pas absents, à notre connaissance, de la grammaire de Meidinger. Pourquoi ne pas les avoir cités dans cet argumentaire? Quant au type d'exercices qu'on trouve dans Du Vivier au XVI ${ }^{e}$ siècle ou dans Mauger au XVII ${ }^{e}$ siècle, exercices que Chevalier rapprochait en 1968 du type structural et dont Vigner disait en 1984 qu'à « ces tendances près, nous ne sommes pas encore en présence d'exercices au sens où nous l'entendons aujourd'hui ", on pourrait aisément montrer que ces «tendances » étaient présentes, bien avant la fin du XVIII siècle, dans bien d'autres manuels portant sur les langues européennes ( $c f$. Kelly $1976: 101-118^{28}$ ).

\section{De l'émergence de grammaires « nationales »}

Cette troisième partie - qui demanderait à être davantage argumentée - sera aussi notre conclusion. Les supposées "tendances" dont parle Vigner en 1984 sont aussi anciennes en didactique des langues (L2 ou L1) que ce qu'on y appelle de nos jours "grammaire inductive " (des exemples à la règle) opposée à "grammaire déductive " (de la règle aux exemples): Kelly estime que cette opposition existe, sous d'autres formulations, tout au long des vingt-cinq siècles d'enseignement occidental des langues qu'il prend en compte, «mais jamais sur un pied d'égalité $~^{29}$ (ibid. : 34). Que dans la seconde partie du XVIII ${ }^{e}$ siècle on soit passé, entre autres en France, d'un déductif alors dominant dans nombre de collèges à un certain inductif, où l'Émile de Jean-Jacques Rousseau [1762] a sa part, n'est pas contestable. Que cet inductif soit alors une « invention » ou une « construction pédagogique » inédite, sans précédent, l'est bien davantage.

Notre intention, à l'annonce de ce colloque, était d'intervenir sur ce que Kelly (ibid. : 116) estime être «la plus ancienne forme des exercices grammaticaux $»^{30}$, ces chries dont il est question dans les progymnasmata de Quintilien, lequel les entend, dans son premier livre, comme un exercice grammatical et non comme un exercice rhétorique. C'eût été une manière de contester l'argumentaire de ce colloque, en montrant que ce type d'exercices se retrouve depuis les manuels alexandrins de grammaire grecque aux "scénarios » au moyen desquels on "exploitait », en particulier à Mons, les leçons des manuels SGAV de la première génération.

Fin XVIII ${ }^{\mathrm{e}}$ - début XIX ${ }^{\mathrm{e}}$ siècle, il y eut bien un changement dans le statut scolaire de la grammaire quand, selon le sous-titre de la thèse de Chervel (1977), « il fallut apprendre à écrire à tous les petits Français " - y compris à ceux qui ne pratiquaient pas nativement le français. Nous avons vu que Vigner (1984) cite trois courtes phrases de cette thèse, dont la première lie ce changement à «l'apparition d'exercices annexes »; Vigner (2017: 32) n'en re-cite que la seconde et la troisième: "On consultait la grammaire pour apprendre, ou comprendre, la langue. On va désormais se livrer à ces exercices pour apprendre la grammaire. " L'interprétation qu'il fait de ces deux phrases, tant en 1984 qu'en 2017, reprend celle de Chevalier (1968: 385) pour qui 
certains exercices avaient pour objectif, mais dès le $\mathrm{XVI}^{\mathrm{e}}$ siècle, de rendre « inutiles les grammaires ", d'être " un substitut de la grammaire » (cf. aussi supra).

Sans contester cette interprétation, notre thèse est que, fin XVIII ${ }^{e}$ - début XIX ${ }^{e}$ siècle, "se livrer à (d)es exercices pour apprendre la grammaire" s'inscrivait dans une configuration nouvelle non en didactique des langues mais au cœur du savoir des grammairiens pris dans un environnement socio-politique que commençait à dominer «l'idée de nationalité $»^{31}$.

Dumarsais, dans son article Construction (Encyclopédie, vol. 4), oppose la « construction » à la « syntaxe » (ibid. : 73) :

Je crois qu'on ne doit pas confondre construction avec syntaxe. [...] Cicéron a dit selon trois combinaisons différentes, accepi litteras tuas, tuas accepi litteras, \& litteras accepi tuas : il y a là trois constructions, puisqu'il y a trois différens arrangemens de mots ; cependant il n'y a qu'une syntaxe ; car dans chacune de ces constructions il y a les mêmes signes des rapports que les mots ont entr'eux, ainsi ces rapports sont les mêmes dans chacune de ces phrases.

Deux courtes gloses. Ce que Dumarsais dénomme constructions a pour (para)synonymes, dans cette citation, arrangements de mots et phrases, celles-ci étant entendues non au sens qu'on donne à ce terme dans les grammaires actuelles du français, mais au sens qu'il a conservé dans phraséologie: des manières de dire ou des tournures, d'ordre stylistique ou idiomatique, dont Dumarsais dit qu'elles sont jugées vicieuses quand leurs mots « ne sont pas arrangés selon l'usage d'une langue » (ibid.). C'est le sens que phrases a conservé en anglais, langue dans laquelle on l'oppose à sentences.

Seguin (1983) a bien décrit le sinueux et lent processus par lequel les grammairiens du français sont passés, au cours du XVIII ${ }^{\mathrm{e}}$ siècle, des phrases telles qu'ils avaient coutume d'entendre ce pluriel, à ce que Domergue $(1788$ : 85, 193) appelle «la phrase grammaticale ", processus que Seguin considère, selon le sous-titre de son ouvrage, comme essentiel dans «l'histoire du sentiment linguistique français ». En est-il allé de même pour les autres langues européennes? De la syntaxe, telle que l'entendait Dumarsais, on pourrait dire en reprenant le titre de la grammaire de Port-Royal [1660] qu'elle est, contrairement aux phrases tributaires de l'usage de chaque langue, d'ordre général et raisonné, en ce qu'elle transcende par abstraction à la fois la diversité des « arrangement de mots » propres à une langue et la diversité des langues, au moins européennes.

Fin $\mathrm{XVIII}^{\mathrm{e}}$ - début du XIX ${ }^{\mathrm{e}}$ siècle, les grammairiens des langues européennes ont tendance à délaisser Port-Royal, et partant à réduire son " général et raisonné » à une sorte de sous-logique » (celle, par exemple, voulant qu'en français l'indicatif et non le subjonctif suive après que) où l'on ne retrouve guère La Logique ou l'art de penser de PortRoyal [1662]. Ils maintiennent certes une partie de ce que leur a légué la tradition grammaticale gréco-latine, entre autres «les parties du discours », mais ils la prolongent par des notions si ad hoc à la langue qu'ils étudient qu'elles en deviennent peu compréhensibles à qui ne la pratique pas nativement (telles, en français, les notions "article partitif» ou "conditionnel»). Se multiplient alors des grammaires rédigées par des natifs de la langue qu'ils étudient au moyen de cette même et seule langue, grammaires pour ainsi dire franco-françaises, anglo-anglaises, germanoallemandes..., dans lesquelles les grammairiens, oublieux de la distinction qu'avait faite Dumarsais entre les constructions et la syntaxe, ont eu tendance à réduire cette dernière à un ensemble, quelque peu hétéroclite, de constructions ou de structures à même de donner lieu aux exercices tels que les entend l'argumentaire de ce colloque. 
Ce même Dumarsais note sans son article Abstraction de l'Encyclopédie (vol. $1: 46$ ) : «Il n'y a pas hors de nous un être réel qui soit la Grammaire ; il n'y a que des grammairiens qui observent ». Rares ont été, aux XIX ${ }^{\mathrm{e}}-\mathrm{XX}^{\mathrm{e}}$ siècles, les grammairiens à avoir échappé aux contextes « nationalistes » dans lesquels ils ont œuvré.

\section{BIBLIOGRAPHIE}

ARGAUD, Marc \& MARIN, Benoit (1972). « Pédagogies de De vive voix. Deuxième type de pédagogie ». Voix et Images du CREDIF [sans date ni numéro, mars 1972].

BESSE, Henri (1970). « Problème de sens dans l'enseignement d'une langue étrangère ». Langue française, 8, 62-77.

BESSE, Henri \& PORQUIER, Rémy (1984). Grammaires et didactique des langues. Paris : Hatier-Crédif. BOUTON, Charles P. (1972). Les grammaires françaises de Claude Mauger à l'usage des Anglais (XVII siècle). Paris : Éditions Klincksieck.

CHEVALIER, Jean-Claude (1968). Histoire de la syntaxe. Naissance de la notion de complément dans la grammaire française (1530-1750). Genève : Librairie Droz.

CHERVEL, André (1977). Histoire de la grammaire scolaire... et il fallut apprendre à écrire à tous les petits Français. Paris : Payot.

CUQ, Jean-Pierre (dir.) (2003). Dictionnaire de didactique du français langue étrangère ou seconde. Paris : Asdifle-CLE International.

Encyclopédie, ou dictionnaire raisonné des sciences, des arts et des métiers, etc., eds. Denis Diderot and Jean le Rond d'Alembert. University of Chicago : ARTFL Encyclopédie Project (Autumn 2017 Edition), Robert Morrissey and Glenn Roe (eds). En ligne : [http://encyclopedie.uchicago.edu/]. DOMERGUE, François Urbain (1778). Grammaire françoise simplifiée [...]. Lyon : Chez l'Auteur, place de la Comédie.

HÉBRARD, Jean (1982). « L'exercice de français est-il né en 1823 ? ». Études de Linguistique Appliquée, $48,9-31$.

GALISSON, Robert \& COSTE, Daniel (1976). Dictionnaire de didactique des langues. Paris : Hachette.

KELLY, Louis G. (1976) [1969]. 25 Centuries of Language Teaching. Rowley, Mass : Newbury House Pub. QUINTILIEN (1975) [fin I ${ }^{\mathrm{er}}$ siècle]. Institution oratoire. Texte établi et traduit par Jean Cousin. Paris : Les Belles-Lettres, en 2 volumes.

SEGUIN, Jean-Pierre (1993). L'invention de la phrase au XVIII e siècle, contribution à l'histoire du sentiment linguistique français. Paris : Éditions Peeters.

VIGNER, Gérard (1982). «L'exercice en français langue étrangère ». Études de Linguistique Appliquée, 48.

VIGNER, Gérard (1984). L'exercice dans la classe de français. Paris : Hachette. 
VIGNER, Gérard (2017). Systématisation et maîtrise de la langue : l'exercice en FLE. Paris : Hachette.

\section{NOTES}

1. Il avait publié dans ce numéro des ELA un article intitulé «L'exercice en français langue étrangère », dont son ouvrage de 1984 peut être lu comme un développement.

2. Vigner cite cet article dans sa bibliographie (1984: 216), mais il est daté de 1983 au lieu de 1982.

3. Dont l'auteur de ces lignes a rédigé les sept premiers chapitres et Rémy Porquier les quatre autres.

4. Je m'auto-cite afin de ne pas (trop) modifier mes formulations d'il y a plus de trente ans.

5. Le premier article où j'ai tenté de théoriser ce que nous n'appelions pas encore des « exercices de conceptualisation" s'intitulait: "Problème de sens dans l'enseignement d'une langue étrangère » (Besse 1970).

6. Rémy Porquier (Besse \& Porquier 1984 : 255-258) donnait des exemples montrant que les apprenants sont souvent loin de faire un exercice comme l'a prévu son concepteur, usant de raisonnements tactiques pouvant les conduire à trouver la "bonne réponse " par d'autres voies que celle indiquée par la consigne.

7. C'est à la toute fin de son ouvrage qu'on trouve cette remarque: "Une connaissance plus exacte du statut de l'exercice dans la classe de français exigerait en réalité une enquête complémentaire effectuée dans les classes [...], fondée aussi sur l'étude des cahiers d'élèves. » (Vigner 1984 : 212).

8. La « deuxième partie » de l'ouvrage de Vigner (1984) s'intitule: «L'exercice et l'évolution méthodologique».

9. Expression empruntée à Jean Bastuji (1977) : « Pourquoi les exercices de grammaire ?», Langue française, $\mathrm{n}^{\circ} 33$, février : 9 .

10. Ce qui revenait à étendre les principes de la correction phonétique verbo-tonale de Petar Guberina à tous les niveaux du langage, y compris le métalinguistique.

11. Manuel dont les images ne prétendaient pas, contrairement à la plupart des manuels « audiovisuels », visualiser dans des bulles le sens des répliques enregistrées, et dont les «sketches » étaient des "dialogues de situation ", c'est-à-dire des dialogues dont on parvient à deviner les intentions de communiquer des personnages, à l'aide des séquences d'images, sans avoir à en entendre les répliques. L'exemple était celui des énoncés produits par les élèves face à la seconde image de la leçon 15 de ce manuel, leçon où sont introduits pour la première fois les double compléments pronominaux (Alors, Mademoiselle, ce dossier bleu, vous me le donnez ?). On compte, avant que leurs élèves n'écoutent cette réplique, une dizaine d'énoncés visant à formuler l'intention de communiquer d'un patron s'adressant à sa secrétaire, et pas moins d'une trentaine après l'avoir écoutée, dont certains visent à intégrer, mais au seul mode impératif (ce qui permet d'éviter la succession des trois pronoms), ce double complément : Le dossier bleu, apportez-le moi ; Donnez-le moi ; ${ }^{*}$ Apportez-moi le ; etc.

12. Il en va différemment pour l'écrit d'une langue tel qu'il a pu être séculairement façonné, au moins dans la tradition occidentale, par des générations de grammairiens.

13. Ayant moi-même constaté la difficulté que j'avais souvent à trouver la «bonne réponse » attendue par les cacologies du XIX ${ }^{\mathrm{e}}$ siècle.

14. Structuro-Global-Audio-Visuel de Georges Gougenheim, Paul Rivenc et Petar Guberina.

15. Il cite (ibid. : 21) un de nos articles paru dans Langue française en 1980 (« Métalangages et apprentissage d'une langue étrangère ») démontrant que le métalinguistique est inhérent à la classe de L2, même quand on n'y recourt pas au jargon des grammairiens ou des linguistes. 
16. Le mot apparaît (2017:20, où il est question de «la réitération d'une performance »), mais n'est pas un concept opératoire.

17. Nous utilisons la traduction de Jean Cousin (Quintilien 1975).

18. L'abbé Gedoyn, traducteur de l'Institutio oratoria au début du XVIII ${ }^{\mathrm{e}}$ siècle, précise que cet art des chries, sentences, éthologies n'est « pas autre chose qu'une parole remarquable dont on rend raison ".

19. Pour s'en tenir au seul Dictionnaire de l'ancienne langue française et de tous ses dialectes du IXe siècle au XV siècle de Frédéric Godefroy [1891-1902, dix vol.] : exercice, plus rarement exercite, substantif (parfois féminin) y renvoie à l'armée (« levée d'hommes ", "l'exercice des armes »), à la religion (« exercices spirituels»), à « l'exercice » d'un métier ou d'une charge, ou encore à une "période de temps » dans la tenue des comptes, mais non à l'enseignement/apprentissage des langues.

20. Ce que reprennent les cinquième [1798] et sixième [1832-1835] éditions, mais pas la huitième [1932-1935].

21. Mais, dans le Suplemento de son Arte de hablar bien francés [1781] (Sexta impression : 1812, p. 48), Nicolas Chantreau range sous l'étiquette exercicios - exercices ceux qui sont à même de faire un parfait gentilhomme : de la danse au chant, en passant par le saut, la promenade, la course, la chasse, la pêche, l'escrime, le manège et la musique.

22. Le site The ARTFL Encyclopédie (University of Chicago, Robert Morrissey ed.) permet de relever l'ensemble de ses occurrences dans la totalité de l'œuvre : 1754 au singulier, 604 au pluriel.

23. Il s'agit de la citation d'un spécialiste de l'archéologie grecque.

24. Par exemple, aucune occurrence d'exercice(s), pris dans ce sens, aux entrées Grammaire, dont Nicolas Beauzée est le rédacteur principal, et Langue, dont il est le seul rédacteur.

25. Il serait intéressant de voir quels sont ceux, parmi ces quinze sens, qui sont traduisibles dans les autres langues européennes par les équivalents d'exercice dérivés de ce même exercitium (tels exercise, ejercicio, esercizio,...).

26. Notre interprétation de ponctuel dans notre définition de l'exercice en 1984 s'appuyait sur cette partie de la définition de ce dictionnaire.

27. Cf. la $4^{\mathrm{e}}$ édition. En ligne : [http://portail.atilf.fr/cgi-bin/getobject_?p.5:19./

var/artfla/dicos/ACAD_1762/IMAGE/].

28. Ces pages sont consacrées aux Grammar drills qu'il divise en trois types : pattern practice (a list of sentences with identical structure but different words or as the substitution table); the cycle (a description of a series of actions taking place) qu'on trouve chez Gouin mais aussi dans des manuels alexandrins du III ${ }^{\mathrm{e}}$ siècle ; et la chria grammaticale ( $c f$. ci-après).

29. [...] but never on an equal footing.

30. The chria is the most ancien form of grammar drill.

31. Selon le titre d'un ouvrage de Georges Weill : L'Europe du XIX ${ }^{e}$ siècle et l'idée de nationalité. Paris : éditions Albin Michel, 1938.

\section{RÉSUMÉS}

Présentée comme une " évidence », la notion d'exercice de langue n'est pas précisément définie dans l'argumentaire de ce colloque, et l'histoire de ce qui s'y trouve dénommé ainsi ne l'est guère plus. La conception sous-jacente à cette notion a été élaborée dans un numéro des Etudes de 
linguistique appliquée intitulé L'exercice $\left(n^{\circ} 48,1982\right)$ et dans un ouvrage de Gérard Vigner titré L'exercice dans la classe de français (1984) ; la même année que Grammaires et didactique des langues d'Henri Besse \& Rémy Porquier dont la conception des « exercices grammaticaux » est différente. Notre première partie rappelle et confronte ces deux conceptions : une proche de la linguistique appliquée, qui fait de l'exercice une activité spécifique ; une s'inscrivant dans la réémergence de la didactique des langues où les exercices visent à apprendre plus qu'à enseigner une langue. C'est cette conception que notre seconde partie cherche à justifier en rappelant quel a été l'historique du mot exercice(s), depuis son origine gréco-latine à nos dictionnaires spécialisés actuels, en passant par les dictionnaires généraux français des XVIIe-XIXe siècles et l'Encyclopédie. D'où une conclusion à discuter : s'il y a eu une innovation en matière d'exercices de langue à la fin du XVIIIe siècle, elle est liée à l'abandon d'une grammaire générale et raisonnée au profit de grammaires qui, réduisant la syntaxe à un ensemble de constructions supposées propres à chaque langue, deviennent " nationales».

Presented as self-evident, language exercises as a notion are not defined precisely in the present conference's argument, and the history of what is thus named is nowhere explained more precisely. Our hypothesis is that the concept underlying the notion was set forth, in the case of French and France in an issue of Études de linguistique appliquée entitled L'exercice $\left(n^{\circ} 48,1982\right)$ and in Gérard Vigner's book L'exercice dans la classe de français published in 1984 the same year as Grammaires et didactique des langues by Henri Besse and Rémy Porquier who had a different conception of "grammatical exercises". In our first part we return to and compare these two conceptions: one close to applied linguistics, according to which the exercise is a specific activity, while the other stems from the revival of language didactics and exercises then aim at learning rather than teaching a language. It is the latter conception which, in our second part, we try to justify by referring to the history of the word exercice(s), from its Greco-Latin origin to the present specialized dictionaries, via the French general dictionaries of the 17th-18th centuries and L'Encyclopédie. Hence our conclusion to be discussed: if there was any innovation in the field of language exercises at the end of the 18th century, it was the consequence of the dismissal of a general and reasoned grammar in favour of grammars which by reducing the syntax to a set of constructions supposedly specific to every language, become "national".

\section{INDEX}

Mots-clés : histoire, exercice(s), langues, linguistique appliquée, didactique des langues

Keywords : history, exercise(s), languages, applied linguistics, languages didactics

\section{AUTEUR}

HENRI BESSE

ENS de Lyon hebesse@laposte.net 